\title{
A systematic review of assessment approaches to predict opioid misuse in people with cancer
}

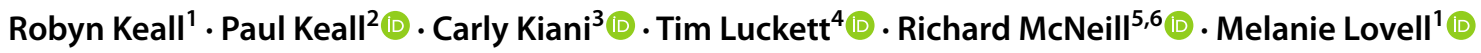

Received: 13 August 2021 / Accepted: 2 February 2022 / Published online: 15 February 2022

(c) The Author(s) 2022

\begin{abstract}
Context Cancer prevalence is increasing, with many patients requiring opioid analgesia. Clinicians need to ensure patients receive adequate pain relief. However, opioid misuse is widespread, and cancer patients are at risk.

Objectives This study aims (1) to identify screening approaches that have been used to assess and monitor risk of opioid misuse in patients with cancer; (2) to compare the prevalence of risk estimated by each of these screening approaches; and (3) to compare risk factors among demographic and clinical variables associated with a positive screen on each of the approaches. Methods Medline, Cochrane Controlled Trial Register, PubMed, PsycINFO, and Embase databases were searched for articles reporting opioid misuse screening in cancer patients, along with handsearching the reference list of included articles. Bias was assessed using tools from the Joanna Briggs Suite.

Results Eighteen studies met the eligibility criteria, evaluating seven approaches: Urine Drug Test (UDT) $(n=8)$; the Screener and Opioid Assessment for Patients with Pain (SOAPP) and two variants, Revised and Short Form $(n=6)$; the Cutdown, Annoyed, Guilty, Eye-opener (CAGE) tool and one variant, Adapted to Include Drugs $(n=6)$; the Opioid Risk Tool $(\mathrm{ORT})(n=4)$; Prescription Monitoring Program (PMP) $(n=3)$; the Screen for Opioid-Associated Aberrant Behavior Risk (SOABR) $(n=1)$; and structured/specialist interviews $(n=1)$. Eight studies compared two or more approaches. The rates of risk of opioid misuse in the studied populations ranged from 6 to $65 \%$, acknowledging that estimates are likely to have varied partly because of how specific to opioids the screening approaches were and whether a single or multi-step approach was used. UDT prompted by an intervention or observation of aberrant opioid behaviors (AOB) were conclusive of actual opioid misuse found to be 6.5-24\%. Younger age, found in 8/10 studies; personal or family history of anxiety or other mental ill health, found in 6/8 studies; and history of illicit drug use, found in 4/6 studies, showed an increased risk of misuse.

Conclusions Younger age, personal or familial mental health history, and history of illicit drug use consistently showed an increased risk of opioid misuse. Clinical suspicion of opioid misuse may be raised by data from PMP or any of the standardized list of AOBs. Clinicians may use SOAPP-R, CAGE-AID, or ORT to screen for increased risk and may use UDT to confirm suspicion of opioid misuse or monitor adherence. More research into this important area is required.

Significance of results This systematic review summarized the literature on the use of opioid misuse risk approaches in people with cancer. The rates of reported risk range from 6 to $65 \%$; however, true rate may be closer to 6.5-24\%. Younger age, personal or familial mental health history, and history of illicit drug use consistently showed an increased risk of opioid misuse. Clinicians may choose from several approaches. Limited data are available on feasibility and patient experience. PROSPERO registration number.
\end{abstract}

CRD42020163385.

Keywords Cancer $\cdot$ Opioid $\cdot$ Pain $\cdot$ Screening $\cdot$ Drug misuse

This research was undertaken to assist in the completion of the Cancer Pain Guidelines for the NSW Cancer Council.

Robyn Keall

rkeall@hammond.com.au

Extended author information available on the last page of the article

\section{Introduction}

\section{Cancer as a chronic disease}

Cancer diagnoses worldwide are increasing, with over 18 million new cases and over 9 million deaths in 2018 [1]. 
Whilst continuing to be the second highest cause of death, thanks to new treatment options, the death rate has dropped by $24 \%$, with 7 out of 10 people surviving for 5 years from diagnosis [2].

\section{Cancer pain}

Pain affects $39 \%$ of people with potentially curable cancer, $55 \%$ of people whilst undergoing curative or palliative treatment, and $66 \%$ of those with advanced and or metastatic cancer [3]. Opioids are the pharmacological treatment of choice for cancer pain that is not responsive to simple analgesics [4].

\section{Opioid misuse}

Opioid misuse or non-medical use is defined as use of a legally prescribed drug for euphoric rather than analgesic effect, using it in a manner or strength that has not been prescribed or using someone else's prescribed drug (i.e., "diverting" it) [5].

\section{Opioid prescription and misuse}

The extension of opioids for use in chronic non-cancer pain (CNCP) resulted in 168 million opioid prescriptions in the USA in 2018 [6] and a 15-fold increase in opioid prescriptions dispensed in Australia between 1992 and 2012 [7]. The rate of prescription of opioids is increasing in older people with cancer [8]. As legal prescriptions have increased, so have hospitalizations and deaths related to opioids and/or illicit drugs, with 46,802 deaths attributed to prescription opioids alone in the USA in 2018 [9] and approximately 1,000 in 2018 in Australia [10]. It is estimated that $4 \%$ of the USA population over 12 years old and 16 million people worldwide have misused prescription pain medication. Each person who misuses opioids costs USD $\$ 9,000-16,000$ more in healthcare costs compared to non-misusers $[11,12]$. Opioid misuse is a major public health problem created by legitimate prescribers and providers and exacerbated by psychosocial and economic issues [13]. Misuse of prescribed and unprescribed opioids is now one of the major international health crises of the twenty first century [14].

\section{Opioid misuse risk versus opioid misuse}

In this systematic review, we report on both "opioid misuse," which is demonstrated through evidence, and "opioid misuse risk," which can be assessed using a patient questionnaire (directly answered or retrospectively applied from medical documentation) and results in a score which identifies patients as being at high or low risk of opioid misuse.

\section{Opioid prescription misuse and cancer patients}

A recent systematic review of substance use disorder in cancer patients found median rates of $18 \%$ previous or current opioid misuse and $25.5 \%$ alcohol misuse [15]. This higher prevalence of alcohol misuse in cancer patients may reflect a causal pathway, given the metabolites of ethanol are a carcinogen [16]. Risk of opioid misuse can increase with chronicity of treatment [17]. Although the risk of opioid misuse has historically been thought to be low when opioids are prescribed for cancer, there is increasing concern that the risks are, in fact, significant and under-recognized. Concern has led to the development of guidelines for clinicians advising use of harm minimization strategies [4]. However, in a recent survey of palliative care clinicians, most reported having patients with opioid misuse risk and lacked the confidence to adequately manage or mitigate the associated risk, with less than a quarter using any screening tools or strategies [18]. General practitioners may be less concerned about the risk of opioid misuse in cancer patients compared to $\mathrm{CNCP}$, potentially posing a risk due to the under-recognition of this problem [19].

Mitigation strategies are most efficient when employed proportionately to the risk of opioid misuse. Accordingly, this requires a means of screening patients for opioid misuse. Guidance on how to screen patients for opioid misuse should be evidence-based. However, most guidance around screening for clinicians is based on evidence from prescribing for $\mathrm{CNCP}$, and it is unclear how this applies to prescribing for cancer pain.

We conducted a systematic review to address this evidence gap. The aims of this review were (1) to identify screening approaches that have been used to assess and monitor risk of opioid misuse in patients with cancer; (2) to compare the prevalence of risk estimated by each of these screening approaches; and (3) to compare risk factors among demographic and clinical variables associated with a positive screen on each of the approaches.

\section{Methods}

The systematic review was registered with PROSPERO (registration number CRD42020163385) and was performed in accordance with PRISMA guidelines [20].

\section{Eligibility criteria}

In population, intervention, comparator, outcomes, and study (PICOS) terms, inclusion criteria were adolescent and/or adults with a diagnosis of cancer and prescribed or 
considered for prescription of opioids for pain (P); with risk mitigation interventions or strategies to predict or prevent opioid misuse (I); with any or no comparator (C); prevalence of risk for opioid misuse $(\mathrm{O})$; and randomized controlled trials, cohort studies, case-control studies, case series, and audits (S). Articles were included if published in English (or English-translated) in a peer-reviewed journal.

\section{Information sources}

Between March and May 2020, a systematic search was undertaken of the following databases: Medline, Cochrane Controlled Trial Register, PubMed, PsycINFO, and Embase. Reference lists of included articles were searched by hand and eligible articles added.

\section{Search}

The search terms used were "cancer pain," "neoplasm," "cancer patient*.mp" AND "opioid*," "opiate.mp" AND "harm minimization," "risk mitigation," "brief risk questionnaire," "urine drug screen," "SOAPP-R," "ORT," "COMM," "pain medicine questionnaire," "abuse deterrent opioid formulation."

\section{Study selection}

Abstracts were retrieved and duplicates removed. Two independent reviewers (R. K. and C. K.) reviewed all the abstracts, and agreement was made on eligible articles to be retrieved in full. Differences were discussed and with a third and fourth party (R. M. and M. L.), and consensus articles were retrieved in full by R. K. and C. K.

\section{Data extraction}

Data items were extracted by three reviewers (R. M., C. K., and R. K.) including setting, sample characteristics, intervention, research design, comparator, and prevalence of misuse and associated risk factors.

\section{Risk of bias in individual studies}

The reviewers assessed bias using the Joanna Briggs Suite of tools [21].

\section{Synthesis}

Because of heterogeneity between samples and screening approaches, synthesis used a narrative approach [22]. Three authors (R. M, C. K., and R. K.) synthesized the findings of the individual tools in both a tabulated form for numerical comparison and in discussion.

\section{Results}

The searches yielded 98 articles, of which 14 met the criteria and were included, along with four more identified by handsearches $(N=18)$. See Fig. 1 for detail.

\section{Study characteristics}

Of the 18 studies, 14 were case series (8 retrospective), two retrospective cohort studies, one a retrospective case-control study, and one, a case report. There were no randomized controlled trials. All studies were conducted in the USA.

\section{Screening approaches in patients with cancer}

The studies evaluated seven assessment approaches to assess for opioid misuse in patients with cancer: Urine Drug Test (UDT) $(n=8)$; the Screener and Opioid Assessment for Patients with Pain (SOAPP) and two variants, Revised and Short Form $(n=6)$; the Cut-down, Annoyed, Guilty and Eye-opener (CAGE) tool and one variant, Adapted to Include Drugs $(n=6)$; the Opioid Risk Tool (ORT) $(n=4)$;

\section{8 articles found from database search}

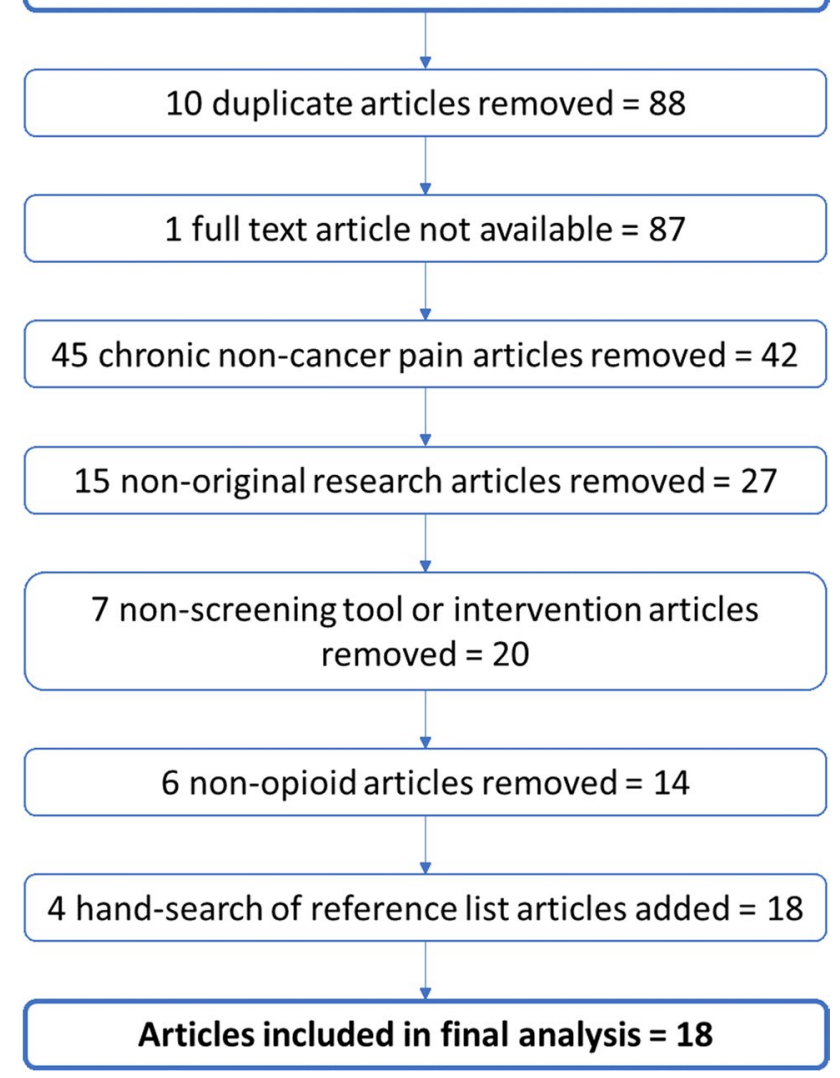

Fig. 1 Flow diagram of the article selection process 
Prescription Monitoring Program (PMP) ( $n=3)$; the Screen for Opioid-Associated Aberrant Behavior Risk (SOABR) $(n=1)$; structured/specialist interviews $(n=1)$; and compared one or more tools $(n=8)$. Thienprayoon et al. (2017) [23] and Anghelescu et al. (2013) [24] both mention the use of a patient interview but did not describe this in any detail, therefore could not be analyzed. The screening approaches that have been used to assess and monitor risk of opioid misuse in patients with cancer are described briefly below.

\section{Screening interventions used in patients with cancer}

\section{Urine Drug Test (UDT)}

UDT can be conducted at the bedside or in the laboratory. Point-of-care UDT can identify the presence or absence of a class of drug and is affordable with a high sensitivity but low specificity [25]. More reliable but also costlier and time-consuming is gas chromatography mass spectrometry. These tests can provide information about specific drugs and their metabolites [26]. False positives can be observed in people with conditions associated with high lactic acidosis, ingestion of poppy seeds, and some medicines, whilst people wishing to avoid detection may refuse, over-hydrate, or add substances to their specimen [26, 27].

Although evidence is limited for UDT to assess risk and monitor opioid treatment, it has been recommended in both the cancer [4] and non-cancer pain population [28]. There are no studies comparing UDT against no UDT as a risk mitigation strategy [29].

\section{The Screener and Opioid Assessment for Patients with Pain (SOAPP)}

The original SOAPP tool consists of 14 questions about behaviors associated with opioid misuse, each scored on a Likert scale from zero ("never") to four ("very often"). A total score of seven or more indicated high risk of opioid misuse. The SOAPP was shown to have adequate sensitivity and specificity [30]. Two revised forms of the SOAPP tool have been developed, both retaining the zero to four Likert scale. Screener and Opioid Assessment for Patients with Pain-Revised (SOAPP-R) was created to improve the sensitivity and specificity of the tool by increasing the number of questions to 24 , with highly significant reliability and predictability [31], whereas the Screener and Opioid Assessment for Patients with Pain-Short form (SOAPP-SF) improved clinical feasibility by reducing the questions to five but also increased the risk of a false positive response by a third [31, 32]. A total score of 18 or more was considered positive for SOAPP-R, and a score of four or more was positive for SOAPP-SF.
The Cut-Down, Annoyed, Guilty, Eye-Opener (CAGE)

The CAGE asks four questions about behaviors associated with alcohol misuse. Answering yes to two or more of the four questions is considered positive. The CAGE is an effective screening tool for alcoholism in many settings with varying success [33-37], with sensitivity between 0.71 and 0.84 and specificity of $0.9-0.95$ [38]. Whilst there is one highly cited study to show daily alcohol use being a risk factor of prescription drug abuse, there is limited evidence elsewhere to support this claim [39, 40]. This limited evidence for use of CAGE to identify opioid misuse risk has led to the development of the Cut-Down, Annoyed, Guilty, Eye-Opener-Adapted to Include Drugs (CAGE-AID), first validated in 1995 [41] and found to be more sensitive but less specific for substance misuse than the CAGE [42]. The CAGE-AID has expanded the 4 CAGE questions to include drug use in the questions, i.e., "Have you ever you felt the need to Cut down on your drinking or drug use?" Answering yes to one or more questions is considered positive and further evaluation should be undertaken. The questions refer to a person's lifetime usage and drug use refers to both illicit and misuse of legal drugs.

\section{The Opioid Risk Tool (ORT)}

The ORT consists of 10 items, resulting in a score from 0 to 24. Patients are assessed as low (0-3), moderate (4-7 s), or high risk $(>8)$. Categories are personal or family history of substance abuse, age, history of pre-adolescent sexual abuse, and a history of specific psychiatric disorders. Although validated in chronic non-cancer pain, a recent systematic review concluded that the ORT did not predict aberrant drug behavior in chronic pain due to the low quality of the included studies [43]; another paper found inconsistency in results with sensitivity ranging from 0.20 to 0.99 and specificity from 0.16 to 0.88 [29]. The ORT has not been validated in the cancer population.

\section{Prescription Monitoring Program (PMP)}

PMPs are databases which record details, from a variety of sources, about prescriptions including the prescriber, the medication, and the recipient. PMPs can alert prescribers in real time or be searched to obtain information. PMPs are not universally available. In a recent systematic review of the effectiveness of PMPs, the authors found limited evidence of reducing opioid prescribing, dispensing, and multiple prescribers [44]. 


\section{Screen for Opioid-Associated Aberrant Behavior Risk (SOABR)}

The SOABR tool was intended for use in adolescents and young adults with cancer [24]. It assesses the presence or absence of six risk factors, including substance misuse, mental health diagnoses, and a history of sexual abuse. As this tool has not been validated to date, interpretation is less established than for other tools.

Table 1 summarizes the included studies' designs, settings, sample characteristics, screening intervention(s), findings regarding the prevalence of opioid misuse, and comments. The studies are grouped by screening approach. Rates of increased opioid misuse risk in the studied populations ranged from 6 to $65 \%$.

\section{Urinary Drug Test}

Of the included studies, eight used UDT to screen for or identify opioid misuse based on clinical suspicion raised by observation of aberrant opioid behaviors (AOBs) or positive result on screening tool $[23,24,45-50]$. Four studies used targeted UDT [47, 49-51], two studies used UDT for all patients [23, 48], one reviewed a screening tool against previously recorded but motivation unknown UDT [46], and one study compared targeted UDT to random UDT [45]. In the reviewed studies, UDTs were more commonly abnormal due to the presence of illicit or nonprescribed opioids rather than the absence of a prescribed drug. For this review, only data for inclusion of unprescribed opioids was considered as evidence of opioid misuse; this was defined in 5 studies [45-47, 49, 50] and 1 case study [51]; we consider that although the absence of a prescribed opioid may point to diversion, it may reflect patient choice. Therefore, the actual rates of opioid misuse shown by UDT in the five studies ranged from 6.5 to $24 \%$, aggregate of $98 / 652(15 \%)$.

Of the included studies, UDT was used for primary screening as part of usual practice $[23,45,46]$ and/or secondary screening after risk identified by (i) another tool ORT [50], CAGE [47], CAGE-Adapted to Include Drugs (CAGE-AID) [51], or (ii) clinical suspicion based on history of drug misuse and/or aberrant opioid behaviors and/ or high symptom expression on validated tool [45, 47, 49-51].

Clinical suspicion was the reason for the majority of the UDT. Koyyalagunta et al. (2018) screened both opioid-naïve and opioid-familiar patients referred by other clinicians as routine practice before prescribing opioids [46]. Arthur et al. (2020) explored the strength of clinical suspicion over random selection in their cohort study, finding an almost twofold increase in abnormal UDTs [45].

\section{Screener and Opioid Assessment for Patients with Pain (SOAPP)}

Seven included studies used one of the forms of the SOAPP tool as a screening intervention for patients with cancer [24, 46, 48, 49, 52-54]. Most were case series apart from one retrospective cohort study.

Yennurajalingam et al. (2018) studied 729 adults in the context of comparing the CAGE-AID to the validated SOAPP. One hundred forty-three out of 729 (19.6\%) participants were found to have high risk per SOAPP [52].

Three studies used the SOAPP-SF [46, 49, 53]. Childers et al. (2015) conducted a prospective study in a palliative care clinic using SOAPP-SF and found that $46 \%$ of patients had a positive score. Over $90 \%$ of patients had a cancer diagnosis, but just over a quarter had non-cancer pain. Those with non-cancer pain were more likely to also complete a UDT and account for $75 \%$ of the abnormal UDT results. Though the abnormal UDTs were further classified to show unexpected opioids present, there was no statistically significant findings to understand those patient characteristics. The authors did not discuss any correlation between the two screening tools [49].

SOAPP-SF was also used in two retrospective chart reviews by the same lead author. Koyyalagunta et al. (2013) reported the findings from over 500 patients at a cancer pain clinic in which the SOAPP-SF is integrated into usual practice and 13\% had a history of illicit drug use (IDU) [53]. SOAPP-SF indicated $29 \%$ of patients were high risk. Of their second case series of 167 patients who were also subjected to UDT, 65\% (97/167) were abnormal [46]. Abnormal UDT was further defined to those with unexpected opioid inclusions 36/167 (22\%) and all abnormal UDTs correlated with positive SOAPP-SF, in particular the question "how often have you used illegal drugs in the last 5 years?" The authors posit that this direct question alone may be adequate to stratify risk in this population [46].

Two case series used SOAPP-R. Anghelescu et al. (2013) presented a small case series of young adults from a specialist pain center. Thirteen patients completed the SOAPP$\mathrm{R}$, and this was combined with a psychologist assessment. Seven of these 13 patients were classified as high risk using both the psychologist assessment and SOAPP-R [24]. Yasin et al. (2019) describe their findings of 69 adults with cancer who completed the SOAPP-R; some underwent UDT based on clinical suspicion, and some had documentation of AOBs. The authors describe AOBs and found the SOAPP-R to have a sensitivity of 0.75 and specificity of 0.80 for opioid misuse [48].

A larger, prospective series was presented by ReyesGibby et al. (2016), who used the SOAPP-R to screen 209 patients with cancer already receiving opioids and attending the emergency department. One hundred ninety-eight out of 


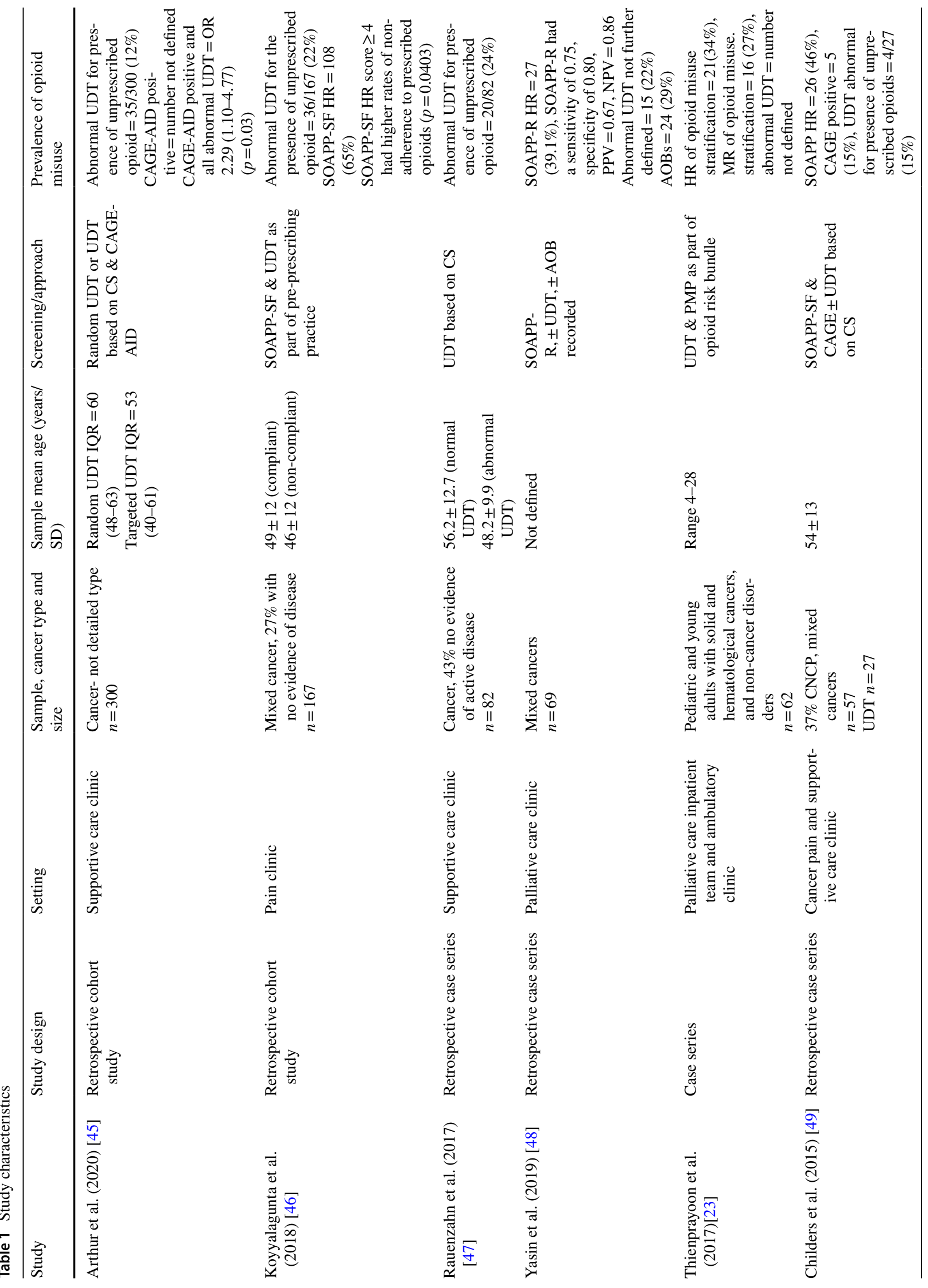




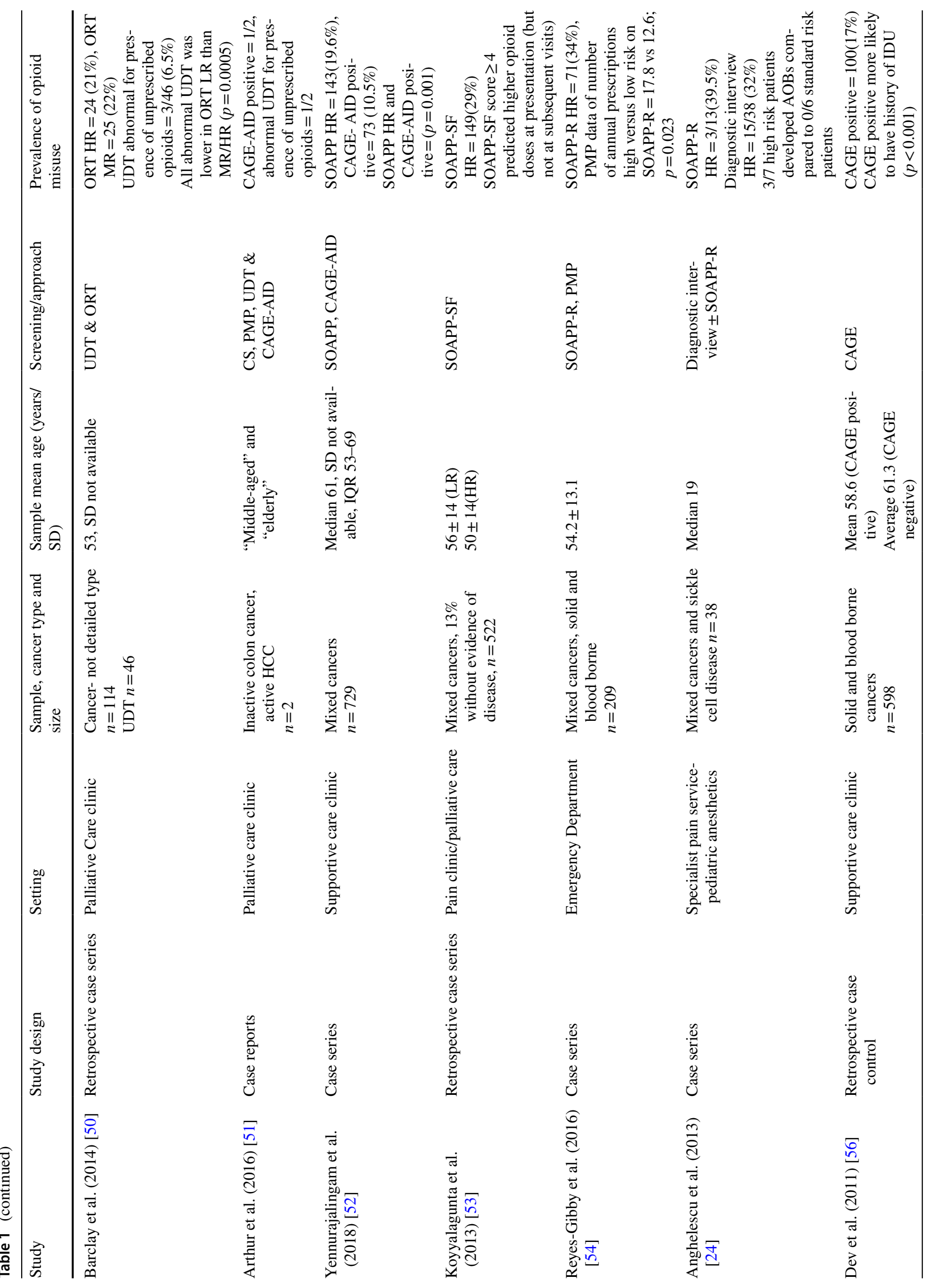




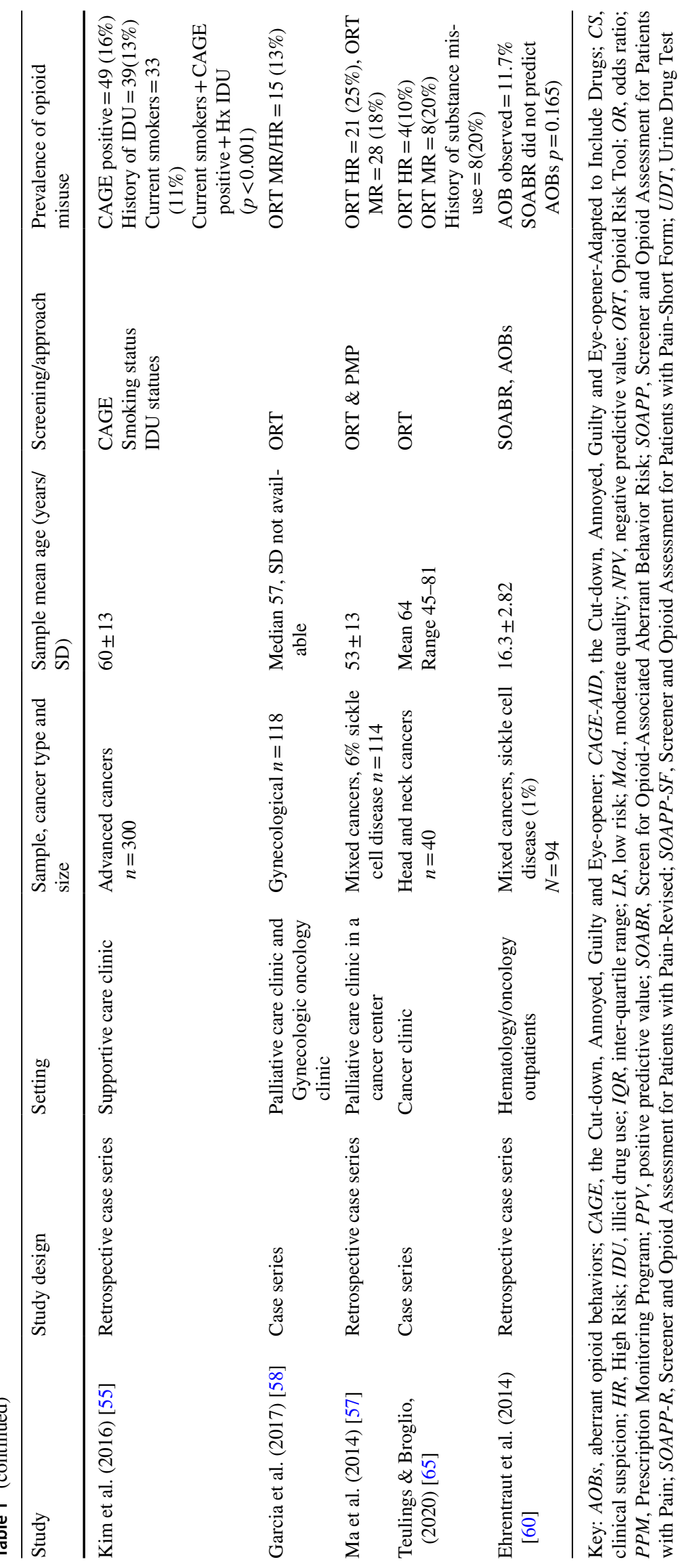


209 had their PMP data reviewed. Thirty-three percent of patients were classified as high risk using the SOAPP-R, and those with a positive SOAPP-R had received more prescriptions as recorded on the state-wide PMP $(p=0.023) .14 .8 \%$ of patients had a history of IDU, and 3.8\% currently used illicit drugs. This study also concluded that the SOAPP-R with its 24 questions was feasible [54].

\section{CAGE and CAGE-AID}

Mixed results were found in the included studies, from no correlation between CAGE positive and abnormal UDT [49] to statistically significant correlation with smoking history [55] and/or illicit drug use through history taking [55, 56].

Arthur et al.'s (2020) cohort study used CAGE-AID and clinical suspicion to conduct UDT, finding a CAGE-AID positive correlated with abnormal UDT [45]. However, it is not clear whether CAGE-AID results alone were enough to prompt a UDT. Yennurajalingam et al. (2018) compared CAGE-AID to SOAPP and found a sensitivity of $43.9 \%$ and specificity of $90.9 \%$ and positive predictive value of $53.2 \%$ [52].

\section{Opioid Risk Tool (ORT)}

Four studies used the ORT in patients with cancer. Two studies, Barclay et al. (2014) and Ma et al. (2014), retrospectively reviewed the charts of cancer patients and found an average of $21.5 \%$ for both moderate and high risk of opioid misuse [50, 57]. Barclay et al. (2014) had results of 46/114 participants who had also had a UDT. Abnormal UDT were present in $62.5 \%$ of those stratified as medium to high risk but also $7 \%$ of the $57 \%$ of total participants assessed as low risk. After corroboration of the ORT by the UDT, Barclay et al. (2014) found family history of alcohol misuse and personal history of IDU were most predictive of opioid misuse [50].

The remaining two studies were a prospective case series and a pilot feasibility study in narrower groups of cancers, gynecological and head and neck, respectively. Garcia et al. (2017) surveyed women with gynecological cancers with the lowest prevalence of all the included studies. Only $7 \%$ were classified as moderate and $6 \%$ as high risk, those with cervical cancer more likely than most to be high risk [58]. Whilst Teulings et al. (2020), studied the ORT in those with head and neck cancers, a population were associated with higherthan-average alcohol and smoking rates though not necessarily opioid misuse, and $20 \%$ were classified as moderate risk and 10\% high [58]. The strongest predictors of scoring high risk on ORT were depression, family or personal alcohol misuse, younger age, and smoking, though this may reflect the subgroup of head and neck cancer patients that are associated with tobacco and alcohol use [59].

\section{Prescription Monitoring Program (PMP)}

Reyes-Gibbs et al. (2016) used their state PMP to compare with the results of the SOAPP-R, finding statistically significant correlation with high SOAPP-R scores and higher than expected number of prescriptions and prescribers [54]. Arthur et al. (2016) used PMP data and UDT to confirm suspicion of AOBs [51].

\section{Screen for Opioid-Associated Aberrant Behavior Risk (SOABR)}

Anghelescu et al. (2013) used correlation between AOB and both the presence of any risk factor, and the number of risk factors present to create the SOABR [24].

The SOABR tool has only been used in a single study to date, a retrospective case series of 94 patients with an oncological or hematological malignancy. Ehrentraut et al. (2014) report 10 of the 11 patients who demonstrated AOB scored at least one point using the SOABR tool [60].

\section{Clinical suspicion}

Clinical suspicion of opioid misuse was mentioned in several studies. What raises clinical suspicion was not fully explained in all of the studies but may include issues such as $\mathrm{AOB}$, including requests for lost prescriptions, missing appointments, requests for escalations, telephone calls for frequent refills $[46,47,51]$ patients without active cancer or with non-cancer pain [49], and history of drug misuse [47, 50]. Yasin et al. (2019) propose a separate list of five AOBs, but of note, one of these includes a history of drug misuse which could be considered a risk factor rather than a current AOB [48]. Neither list has been definitively validated, although the 17-point list has a clear derivation reported based on literature of adults with chronic non-cancer pain. See Table 2 for a list of AOB based on the literature [24, $48,60]$.

\section{Correlations between screening tools and other factors in identifying opioid misuse risk}

Three factors have the capacity to provide definitive evidence of opioid misuse: AOB, PMP, and UDT. Three studies correlated a screening tool with AOBs. In one small study, SOAPP-R combined with a specialist psychology review was associated with a higher rate of AOBs, but no statistical analysis was conducted [24]. In another study, SOAPP-R had a specificity of 0.75 and sensitivity of 0.80 when compared to a combination of AOB and UDT [48]. A study comparing SOABR and AOBs found no statistically significant correlation [24]. PMP data was correlated with the SOAPP-R tool in a single study; higher SOAPP-R 
Table 2 Aberrant opioid behaviors compiled from included articles

\begin{tabular}{ll}
\hline Category & Behavior example \\
\hline Observable behaviors & $\begin{array}{c}\text { Not keeping appointments, requesting scripts from multiple prescribers, requests for scripts via phone, claiming } \\
\text { lost or stolen scripts, pill count irregularities, asking for drug by street name, distress if drug unavailable, resist- } \\
\text { ance to change plan }\end{array}$ \\
$\begin{array}{l}\text { Medication non-compliance } \\
\text { Interpersonal behaviors }\end{array}$ & $\begin{array}{l}\text { Self-increasing dose or frequency, self-medicating for non-analgesic effect including euphoria, anxiety, and sleep } \\
\text { Hoarding drugs, concerns by caregivers and/or family, decreased level of functioning, requiring drug to be able to } \\
\text { function }\end{array}$ \\
Illegal behaviors & Illicit drug use, stealing, selling, or forging prescriptions, sourcing drugs illegally \\
\hline
\end{tabular}

scores were associated with more frequent opioid prescriptions [54]. UDT has been correlated with CAGE-AID [45], SOAPP-SF [46], and the ORT [50]. There was significant heterogeneity in these studies in abnormal UDT results, including the presence of unprescribed opioid, the absence of prescribed opioid, and the presence of illicit non-opioid drugs. Two further studies measured clinical outcomes. One measured AOBs but did not correlate these with the CAGE and SOAPP-SF results, perhaps due to the small sample size [49]. Another found that high SOAPP-SF scores correlated with high opioid doses at initial presentation to their pain clinic, but the correlation was not maintained at subsequent visits and is not an established marker of opioid misuse [53].
The articles identified varied in the weight that certain factors convey to increase clinical suspicion. Younger age, found to be significant in $8 / 10$ studies, personal or family history of anxiety or other mental ill health, found to be significant in $6 / 8$ studies, and history of illicit drug use, found to be significant in 4/6 studies, showed an increased risk of misuse. See Table 3 for factors identified as increasing risk.

\section{Discussion}

This systematic review of assessment approaches to predict opioid misuse in people with cancer summarizes the existing research on screening for opioid misuse in patients

Table 3 Demographic and clinical factors identified by included articles as associated with risk of opioid misuse

\begin{tabular}{|c|c|c|}
\hline $\begin{array}{l}\text { Risk factor identified to increase risk of opioid } \\
\text { misuse }\end{array}$ & $\begin{array}{l}\text { Approaches used from included articles show- } \\
\text { ing statistical significance } p<.05\end{array}$ & $\begin{array}{l}\text { Approaches used from included articles not } \\
\text { showing statistical significance } p<0.05\end{array}$ \\
\hline Younger age & $\begin{array}{l}\text { UDT [45-47], SOAPP-SF [46, 49, 52, 53], } \\
\text { CAGE [56] }\end{array}$ & ORT $[58,57]$ \\
\hline $\begin{array}{l}\text { Personal or family history of anxiety or other } \\
\text { mental ill health }\end{array}$ & $\begin{array}{l}\text { UDT [45-47, 52], SOAPP-SF [53], SOAPP-R } \\
\text { [54] }\end{array}$ & SOABR [60], ORT [57] \\
\hline History of IDU & ORT [50], CAGE [56, 55], SOAPP-R [54] & ORT[57], UDT [46] \\
\hline Gender (male) [46] & CAGE [56], UDT [45], ORT [50] & $\begin{array}{l}\text { SOABR [60], UDT [46], SOAPP-SF [53], ORT } \\
\text { [57] }\end{array}$ \\
\hline Active use nicotine & CAGE $[56,55]$ & ORT $[58,57]$ \\
\hline History of alcohol use & CAGE [55] & UDT[47], ORT [57] \\
\hline Cancer type or treatment status & CAGE [56] & $\begin{array}{l}\text { ORT[58], SOAPP-SF 29,[53], UDT [46], ORT } \\
\text { [57] }\end{array}$ \\
\hline Concurrent use of two or more opioids & SOABR [60] & \\
\hline Single, divorced, or never married & & ORT [57] \\
\hline $\begin{array}{l}\text { History of pre-adolescent sexual abuse } \\
\text { (women) }\end{array}$ & & ORT [57], SOABR [60] \\
\hline Family history of alcohol use & & ORT [50], SOABR [60], ORT [57] \\
\hline MEDD & UDT [45] & SOAPP-SF [53], UDT [47], CAGE [56] \\
\hline Expression of pain/symptoms & SOAPP-SF $[52,53]$ & $\begin{array}{l}\text { SOAPP-SF [49], CAGE[46, 49, 58], ORT[57], } \\
\text { UDT [46] }\end{array}$ \\
\hline Race & UDT[47] & CAGE [56] \\
\hline Socioeconomic status & SOAPP-SF [53] & UDT [46] \\
\hline
\end{tabular}

Key: $C A G E$, the Cut-down, Annoyed, Guilty and Eye-opener; $I D U$, illicit drug use; $M E D D$, morphine equivalent daily dose; $O R T$, Opioid Risk Tool; SOABR, Screen for Opioid-Associated Aberrant Behavior Risk; SOAPP-R, Screener and Opioid Assessment for Patients with PainRevised; SOAPP-SF, Screener and Opioid Assessment for Patients with Pain-Short Form; UDT, urine drug test 
with cancer. Seven approaches have been identified. Four are clinical tools, SOAPP, ORT, SOABR, and CAGE (and their variants), used to identify risk factors; the remainder are dedicated specialist assessments or interviews and PMP and UDT which can confirm misuse. A high percentage (average $45 \%$ ) of the illicit drug detected in the abnormal UDT was cannabis; in many cases, it was the only aberration, and in some geographical regions, cannabis may be decriminalized or legally prescribed. Rates of opioid misuse risk from the included studies ranged from 6 to $65 \%$ with confirmed opioid misuse through UDT (presence of non-prescribed opioid only was $6.5-24 \%$ [24, 45, $46,48,50,53,60]$. SOAPP-R, ORT, and CAGE-AID were more reliable than CAGE, SOAPP, SOABR, and SOAPPSF in identifying increased risk as confirmed by AOB, PMP, or UDT. Younger age, personal or familial mental health history, and history of illicit drug use consistently showed an increased risk of or actual opioid misuse. Clinical suspicion may be raised by any of the list of aberrant opioid behaviors drawn from the literature. UDT, AOBs, and PMP data may be used to confirm clinical suspicion and/or monitor adherence to treatment.

The only two studies to report the rate of AOBs in their whole population both report rates of $10-15 \%$, although these studies were both small and limited to a specific population of adolescents and young adults with specialist cancer pain [24, 60]. Two later studies from the same center, identified during the peer review process and so not included in this review, provide early evidence of a similar rate in adults with cancer. One used universal screening for AOBs and found a prevalence of $19 \%$ [61], although a single AOB may not reflect true opioid misuse. The second used random UDT and found 15\% had an abnormality when cannabis was excluded, although other illicit drugs were included [62]. In both studies, SOAPP and CAGEAID were statistically correlated with the presence of an $\mathrm{AOB}$, but positive and negative predicative values were not reported. The two studies in adolescents and both adult studies were conducted in single centers and all in the USA [24, 60-62]. Coupling AOB rates with abnormal UDT rates from the included studies suggest the rates of opioid misuse in the cancer patient population to be closer to $15 \%$; however, this rate comes from a small number of studies. It would be useful to confirm these findings in other centers and countries. Data from screening questionnaires suggest risk factors are prevalent in the cancer population, and between 6 and $65 \%$ of patients are classified as high risk. This large variation suggests screening questionnaires likely overestimate the true risk of opioid misuse in this population. A 2015 systematic review of opioid misuse risk in the chronic non-cancer pain population also found wide ranges of results from $<1$ to $81 \%$ of increased risk [63].
The rates of opioid misuse identified by targeted UDT were high in all studies. The rates were similar whether UDT was ordered based on clinical suspicion alone or using a dedicated screening tool. There is insufficient evidence to support the routine use of screening interventions other than UDT. There is weak evidence to support the universal use of UDT to identify opioid misuse. However, UDT will only identify patients with established misuse and allow mitigation of harm to themselves or others rather than identifying patients at risk to prevent harm. The absence of prescribed drug raises concerns for diversion of drug: the bulk of people who take opioids not prescribed for them have been obtained from a friend or relative at no cost. These are generally entry-level misusers, whilst those that continue to misuse over a sustained period will likely source their opioids through drug dealer or physicians [64]. Screening for drug misuse and/or mental health in the home, as reported in one study, may be helpful in stratifying risk [23].

Several research gaps in today's evidence have emerged from this review. Included studies were conducted in the USA alone. It is unclear whether other countries have similar rates of opioid risk. Future research should focus on correlating the results of screening interventions such as UDT and AOBs, with established diagnoses of opioid misuse. Feasibility studies are required to support the implementation of routine screening for opioid misuse from patient, clinician, and healthcare system perspectives. The psychosocial impact of both screening and subsequent management strategies on patients and clinicians is crucial to understanding the holistic impact of screening and risk mitigation strategies. More work is needed to understand whether, and how, identification of actual opioid misuse or assessed risk alters patient management.

There is more research into opioid misuse risk in the chronic non-cancer pain setting than cancer pain setting with existing practice guidelines [4]. For example, the current opioid misuse measure (COMM) tool is recommended to screen for opioid misuse in patients already receiving opioid therapy; however, there were no studies of the COMM in the cancer population identified by this review. There is no research to understand the psychosocial effects of screening and management of opioid misuse in cancer patients.

This systematic review had several limitations including the narrow search criteria to include only studies with active cancer diagnoses published in a peer-reviewed English language journal. The heterogeneity of the studies made comparative analysis challenging including detailing the reliability of some of the tools. 


\section{Conclusions}

This review found that the risk of opioid misuse varies between 6 and $65 \%$ depending on the tool used and the population screened, with rates of documented opioid misuse closer to $15 \%$. Younger age, personal or familial mental health history, and history of illicit drug use consistently showed an increased risk of opioid misuse. Clinicians may choose to use SOAPP-R, ORT, or CAGE-AID to screen for increased risk of opioid misuse. Clinical suspicion of opioid misuse may be raised by data from PMPs if they are available or any of the standardized list of AOBs. Clinicians may use UDT to confirm suspicion of opioid misuse or monitor adherence, but UDT fails to identify those at risk. There is no research to understand the psychosocial effects of screening and management of opioid misuse. There remains an urgent need for further research in this area given the increasing rates of opioid prescription.

Acknowledgements This research was undertaken to assist in the completion of the Cancer Pain Guidelines for the NSW Cancer Council.

Author contribution COI declarations have been completed for all authors.

Funding Open Access funding enabled and organized by CAUL and its Member Institutions. The authors would like to thank the Friends of the Mater Hospital, Sydney, Australia, for the financial support to conduct this review.

Availability of data and material Data are available, and method of accessing the same is transparent.

Code availability Not applicable.

\section{Declarations}

Ethics approval Not applicable.

Consent to participate Not applicable.

Consent for publication Not applicable.

Open Access This article is licensed under a Creative Commons Attribution 4.0 International License, which permits use, sharing, adaptation, distribution and reproduction in any medium or format, as long as you give appropriate credit to the original author(s) and the source, provide a link to the Creative Commons licence, and indicate if changes were made. The images or other third party material in this article are included in the article's Creative Commons licence, unless indicated otherwise in a credit line to the material. If material is not included in the article's Creative Commons licence and your intended use is not permitted by statutory regulation or exceeds the permitted use, you will need to obtain permission directly from the copyright holder. To view a copy of this licence, visit http://creativecommons.org/licenses/by/4.0/.

\section{References}

1. International Agency for Research on Cancer- World Health Organisation. Global Cancer Observatory,. 2018 [cited 2020 4th November,]

2. Australian Institute of Health and Welfare, Cancer in Australia: In brief 2019. Cancer series no. 122. , A.I.o.H. Wellbeing, Editor. 2019: Canberra.

3. Caraceni A et al (2012) Use of opioid analgesics in the treatment of cancer pain: evidence-based recommendations from the EAPC. Lancet Oncol 13(2):e58-68

4. Swarm RA et al (2019) Adult Cancer Pain, Version 3. 2019, NCCN Clinical Practice Guidelines in Oncology. J Natl Compr Canc Netw 17(8):977-1007

5. National Institute on Drug Abuse. Misuse of prescription drugs report overview. 2021 [cited 2021 9th March,]; Available from: https://www.drugabuse.gov/publications/research-reports/misuseprescription-drugs/overview on 2021

6. Centers for Disease Control and Prevention. U.S. opioid prescribing rate maps,. 2018 March 5, 2020 [cited 2020 4th November 2020]; Available from: https://www.cdc.gov/drugoverdose/maps/ rxrate-maps.html

7. Blanch B, Pearson SA, Haber PS (2014) An overview of the patterns of prescription opioid use, costs and related harms in Australia. Br J Clin Pharmacol 78(5):1159-1166

8. Daniels B, et al. (2020) Patterns of oxycodone controlled release use in older people with cancer following public subsidy of oxycodone/naloxone formulations: an Australian population-based study. Asia-Pacific J Clin Oncol

9. Centers for Disease Control and Prevention. Drug overdose deaths. 2018 March 19, 2020 [cited 2020 4th November]; Available from: https://www.cdc.gov/drugoverdose/data/statedeaths. html

10. Australian Bureau of Statistics, Causes of death Australia. 2018.

11. Reinhart $\mathrm{M}$ et al (2018) The economic burden of abuse of prescription opioids: a systematic literature review from 2012 to 2017. Appl Health Econ Health Policy 16(5):609-632

12. Degenhardt L et al (2014) The global epidemiology and burden of opioid dependence: results from the global burden of disease 2010 study. Addiction 109(8):1320-1333

13. Rummans TA, Burton MC, Dawson NL (2018) How good intentions contributed to bad outcomes: the opioid crisis. in Mayo Clinic Proceedings. Elsevier

14. Volkow ND et al (2019) Addressing the opioid crisis globally. World psychiatry 18(2):231-232

15. Yusufov M, Braun IM, Pirl WF (2019) A systematic review of substance use and substance use disorders in patients with cancer. General Hospital Psychiatry

16. Ratna A, Mandrekar P (2017) Alcohol and cancer: mechanisms and therapies. Biomolecules 7(3):61

17. Roberts AW et al (2020) New-onset persistent opioid use following breast cancer treatment in older adult women. Cancer 126(4):814-822

18. Merlin JS et al (2019) Managing chronic pain in cancer survivors prescribed long-term opioid therapy: a national survey of ambulatory palliative care providers. J Pain Symptom Manage 57(1):20-27

19. Luckett T, et al. (2020) Risk of opioid misuse in people with cancer and pain and related clinical considerations: a Qualitative study of the perspectives of Australian general practitioners. BMJ open 10(2) 
20. Liberati A et al (2009) The PRISMA statement for reporting systematic reviews and meta-analyses of studies that evaluate health care interventions: explanation and elaboration. J Clin Epidemiol 62(10): e1-e34

21. Moola S, M.Z., Tufanaru C, Aromataris E, Sears K, Sfetcu R, Currie M, Qureshi R, Mattis P, Lisy K, Mu P-F (2017) Chapter 7: systematic reviews of etiology and risk . in Joanna Briggs Institute Reviewer's Manual, M.Z. Aromataris E, Editor

22. Popay J, et al. (2006) Guidance on the conduct of narrative synthesis in systematic reviews. A Product from the ESRC Methods Programme. Version 1

23. Thienprayoon R, et al. (2017) Risk stratification for opioid misuse in children, adolescents, and young adults: a quality improvement project. (1098-4275 (Electronic))

24. Anghelescu DL, Ehrentraut JH, Faughnan LG (2013) Opioid misuse and abuse: risk assessment and management in patients with cancer pain. J Natl Compr Canc Netw 11(8):1023-1031

25. Pergolizzi $\mathrm{J}$ et al (2010) The role of urine drug testing for patients on opioid therapy 1(6):497-507

26. Moeller KE, Lee KC, Kissack JC (2008) Urine drug screening: practical guide for clinicians. Mayo Clin Proc 83(1):66-76

27. McDonell MG et al (2016) Utility of point-of-care urine drug tests in the treatment of primary care patients with drug use disorders. J Addict Med 10(3):196-201

28. Chou R (2009) 2009 Clinical guidelines from the American Pain Society and the American Academy of Pain Medicine on the use of chronic opioid therapy in chronic noncancer pain: what are the key messages for clinical practice?. [Review] [66 refs]. 1(7-8):469-77

29. Chou R, et al. (2020) Opioid treatments for chronic pain. Comparative Effectiveness Review No. 229. Agency for Healthcare Research and Quality: Rockville, MD.

30. Butler SF et al (2004) Validation of a screener and opioid assessment measure for patients with chronic pain. Pain 112(1):65-75

31. Butler SF et al (2009) Cross-Validation of a Screener to Predict Opioid Misuse in Chronic Pain Patients (SOAPP-R). J Addict Med 3(2):66-73

32. Butler SF et al (2008) Validation of the revised Screener and Opioid Assessment for Patients with Pain (SOAPP-R). J Pain 9(4):360-372

33. Bernadt MW et al (1982) Comparison of questionnaire and laboratory tests in the detection of excessive drinking and alcoholism. The Lancet 319(8267):325-328

34. Ewing JA (1984) Detecting alcoholism. The CAGE questionnaire. Jama 252(14):1905-1907

35. Saitz R et al (1999) Alcohol abuse and dependence in Latinos living in the United States: validation of the CAGE (4M) questions. Arch Intern Med 159(7):718-724

36. Masur J, Monteiro MG (1983) Validation of the"CAGE" alcoholism screening test in a Brazilian psychiatric inpatient hospital setting. Brazilian journal of medical and biological research 16(3):215-218

37. Zierau $F$ et al (2005) Validation of a self-administered modified CAGE test (CAGE-C) in a somatic hospital ward: comparison with biochemical markers. Scand J Clin Lab Invest 65(7):615-622

38. King M (1986) At risk drinking among general practice attenders: validation of the CAGE questionnaire. Psychol Med $16(1): 213-217$

39. Kwon JH et al (2013) Predictors of long-term opioid treatment among patients who receive chemoradiation for head and neck cancer. Oncologist 18(6):768
40. Chow E et al (2001) Use of the CAGE questionnaire for screening problem drinking in an out-patient palliative radiotherapy clinic. J Pain Symptom Manage 21(6):491-497

41. Brown, R.L. and L.A. Rounds, conjoint screening questionnaires for alcohol and other drug abuse: criterion validity in a primary care practice. Wisconsin medical journal, 1995.

42. Brown RL et al (1998) The prevalence and detection of substance use disorders among inpatients ages 18 to 49 : an opportunity for prevention. Prev Med 27(1):101-110

43. Lawrence R, Mogford D, Colvin L (2015) Systematic review to determine which validated measurement tools can be used to assess risk of problematic analgesic use in patients with chronic pain. (1471-6771 (Electronic))

44. Wilson MN et al (2019) Effectiveness of prescription monitoring programs in reducing opioid prescribing, dispensing, and use outcomes: a systematic review. J Pain 20(12):1383-1393

45. Arthur J et al (2020) Random vs targeted urine drug testing among patients undergoing long-term opioid treatment for cancer pain. JAMA Oncol 6(4):580-581

46. Koyyalagunta D et al (2018) Compliance with opioid therapy: distinguishing clinical characteristics and demographics among patients with cancer pain. Pain Med 19:1469-1477

47. Rauenzahn $\mathrm{S}$ et al (2017) Urine drug screen findings among ambulatory oncology patients in a supportive care clinic. Support Care Cancer 25(6):1859-1864

48. Yasin JT et al (2019) Validity of the screener and opioid assessment for patients with pain-revised (SOAPP-R) in patients with cancer. J Opioid Manag 15(4):272-274

49. Childers JW, King LA, Arnold RM (2015) Chronic pain and risk factors for opioid misuse in a palliative care clinic. Am J Hosp Palliat Care 32(6):654-659

50. Barclay JS, Owens JE, Blackhall LJ (2014) Screening for substance abuse risk in cancer patients using the opioid risk tool and urine drug screen. Support Care Cancer 22(7):1883-1888

51. Arthur JA et al (2016) Aberrant opioid use and urine drug testing in outpatient palliative care. Journal of Palliative Medicine 19(7):1557-7740 ((Electronic))

52. Yennurajalingam $S$ et al (2018) Predicting the risk for aberrant opioid use behavior in patients receiving outpatient supportive care consultation at a comprehensive cancer center. Cancer 124(19):3942-3949

53. Koyyalagunta D et al (2013) Risk stratification of opioid misuse among patients with cancer pain using the SOAPP-SF. Pain Med 14(5):667-675

54. Reyes-Gibby CC, Anderson KO, Todd KH (2016) Risk for opioid misuse among emergency department cancer patients. Acad Emerg Med 23(2):151-158

55. Kim YJ et al (2016) Association between tobacco use, symptom expression, and alcohol and illicit drug use in advanced cancer patients. J Pain Symptom Manage 51(4):762-768

56. Dev R et al (2011) Undocumented alcoholism and its correlation with tobacco and illegal drug use in advanced cancer patients. Cancer 117(19):4551-4556

57. Ma JD et al (2014) A single-center, retrospective analysis evaluating the utilization of the opioid risk tool in opioid-treated cancer patients. J Pain Palliat Care Pharmacother 28(1):4-9

58. Garcia $C$ et al (2017) Prospective screening with the validated opioid risk tool demonstrates gynecologic oncology patients are at low risk for opioid misuse. Gynecol Oncol 147(2):456-459

59. Döbróssy L (2005) Epidemiology of head and neck cancer: magnitude of the problem. Cancer Metastasis Rev 24(1):9-17 
60. Ehrentraut JH et al (2014) Opioid misuse behaviors in adolescents and young adults in a hematology/oncology setting. J Pediatr Psychol 39(10):1149-1160

61. Yennurajalingam S et al (2021) Frequency of and factors associated with nonmedical opioid use behavior among patients with cancer receiving opioids for cancer pain. JAMA Oncol 7(3):404-411

62. Arthur JA et al (2021) Random urine drug testing among patients receiving opioid therapy for cancer pain. Cancer 127(6):968-975

63. Vowles KE et al (2015) Rates of opioid misuse, abuse, and addiction in chronic pain: a systematic review and data synthesis. Pain 156(4):569-576
64. Jones CM, Paulozzi LJ, Mack KA (2014) Sources of prescription opioid pain relievers by frequency of past-year nonmedical use United States, 2008-2011. JAMA Intern Med 174(5):802-803

65. Teulings L, Broglio K (2020) Opioid misuse risk: implementing screening protocols in an ambulatory oncology clinic. Clin $\mathrm{J}$ Oncol Nurs 24(1):11-14

Publisher's Note Springer Nature remains neutral with regard to jurisdictional claims in published maps and institutional affiliations.

\section{Authors and Affiliations}

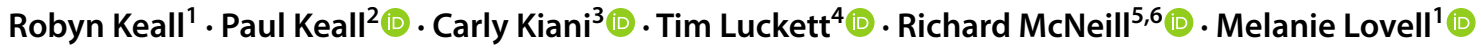

1 HammondCare, Sydney, Australia

2 ACRF Image X Institute Sydney, University of Sydney, Sydney, Australia

3 University of Western Australia, Perth, Australia

4 Faculty of Health, University of Technology, Sydney, Australia
5 Mary Potter Hospice, Wellington, New Zealand

6 Department of Clinical Pharmacology, Christchurch Hospital, Christchurch, New Zealand 